Revue

d'ethnoécologie
Revue d'ethnoécologie

$11 \mid 2017$

Varia + dossier "Cartographie participative" (2)

\title{
Teintures naturelles et teinturiers au Cambodge
}

Natural dyes and dyers in Cambodia

\section{Bernard Dupaigne}

\section{CpenEdition}

Journals

Édition électronique

URL : http://journals.openedition.org/ethnoecologie/2927

DOI : 10.4000/ethnoecologie.2927

ISSN : 2267-2419

\section{Éditeur}

Laboratoire Eco-anthropologie et Ethnobiologie

\section{Référence électronique}

Bernard Dupaigne, «Teintures naturelles et teinturiers au Cambodge », Revue d'ethnoécologie [En ligne], 11 | 2017, mis en ligne le 03 juillet 2017, consulté le 19 avril 2019. URL : http:// journals.openedition.org/ethnoecologie/2927 ; DOI : 10.4000/ethnoecologie.2927

Ce document a été généré automatiquement le 19 avril 2019

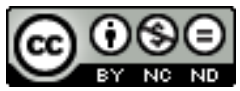

Revue d'ethnoécologie est mis à disposition selon les termes de la licence Creative Commons Attribution - Pas d'Utilisation Commerciale - Pas de Modification 4.0 International. 


\section{Teintures naturelles et teinturiers au Cambodge}

Natural dyes and dyers in Cambodia

Bernard Dupaigne

«Les Khmers teignent eux-mêmes les étoffes qu'ils
fabriquent, à peu près avec les procédés usités
chez nous. Leurs plus belles couleurs sont le rouge,
le jaune et le bleu » (Moura $1882: 337)$. « Ils
utilisent habilement les plantes tinctoriales du
pays, obtenant des couleurs solides, belles, variées,
qui parcourent toute la gamme des teintes »

(Aymonier 1900 : 40)

\section{Introduction}

1 À la fin des années 1960, j’ai passé deux ans au Cambodge, comme enseignant d'Ethnologie à la faculté d'Archéologie de Phnom-Penh. J'y avais consacré tous mes temps libres à la visite des villages, dans la plupart des provinces.

2 Voici ici quelques notes prises sur les procédés de teinture traditionnels. Elles seront reprises dans mon ouvrage en préparation sur les « Tissages de la soie au Cambodge ».

3 Au Cambodge, traditionnellement, les tissus produits étaient teints avec des colorants végétaux ou animaux, cultivés ou trouvés dans la nature, pour donner principalement les couleurs jaune, rouge, bleu-indigo et noire. Ces teintures végétales étaient encore très répandues avant 1975. La teinture des écheveaux de fils, de soie ou de coton, se faisait d'ordinaire au village par les tisserandes elles-mêmes ou par leur mari : les teinturiers spécialisés étaient rares. Il existe encore des teinturières qui préparent les teintures pour les autres tisserandes, en plus de leur propre travail, comme des Khmères de l'île de Koh Sautin. 
Figure 1 : Tisserande khmère, province de Takeo, 1968

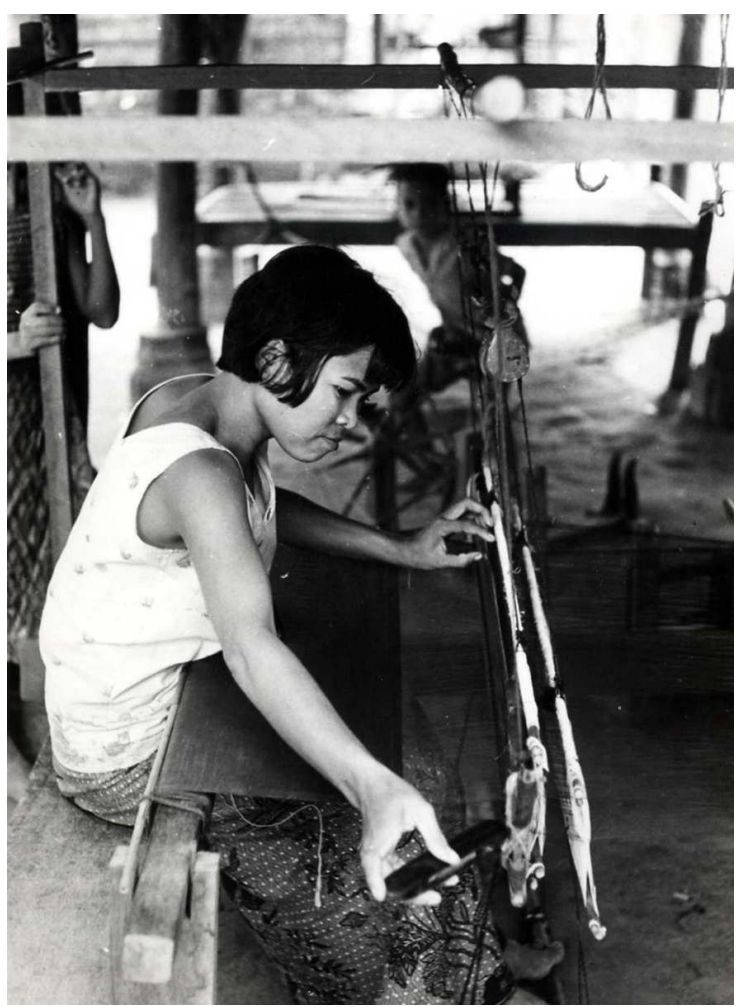

(C) B. Dupaigne

Figure 2 : Tisserande khmère, province de Takeo, 1968

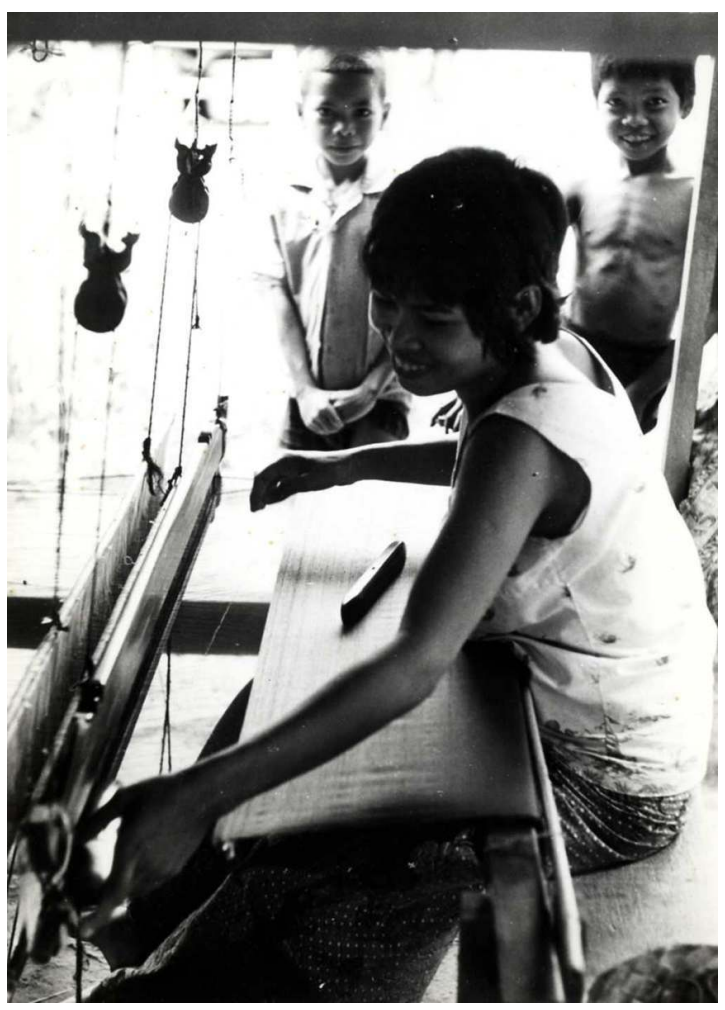

(C) B. Dupaigne

Revue d'ethnoécologie, 11 | 2017 
Figure 3 : Tissage cham, province de Kampot, 1968

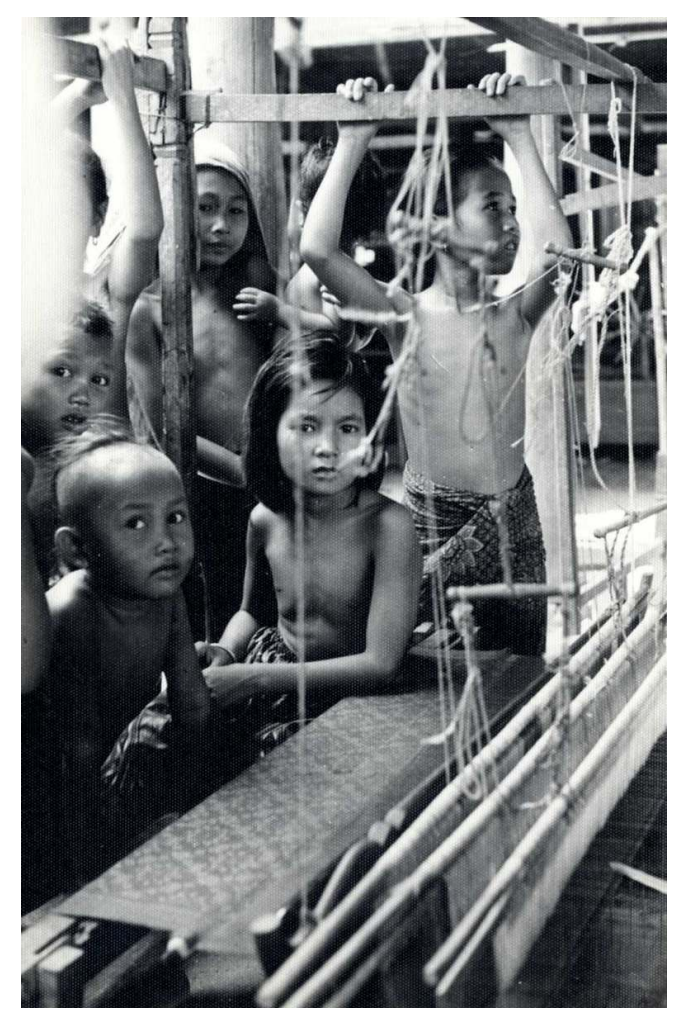

(C) B. Dupaigne

\section{Le processus}

Il faut d'abord blanchir et « décreuser » la soie grège (la soie brute), c'est-à-dire dissoudre la gaine de séricine (grès) qui entoure le fil brut et qui empêcherait la teinture de bien prendre. Pour cela, on prépare une solution de potasse, toeuk (eau) khbong, obtenue par combustion de gousses du kapokier, Bombax malabaricum, sambork kô, un grand arbre présent partout. On lessive des cendres végétales d'écorces de fruits de kapokier, de fruits de bananier, de fleurs de palmiers mâles, ou d'amaranthe (probablement Amaranthus spinosus), phti, et l'on filtre le mélange obtenu (Sek $1973: 32)^{1}$. L'écheveau est plongé « le temps de brûler une baguette d'encens » $-5,10$ ou $20 \mathrm{mn}$ - dans une bassine contenant de l'eau bouillante enrichie de cette solution: un petit verre de khbong pour un meul $(1,5 \mathrm{~kg})$ de soie, soit $60 \mathrm{~m}$ de fil. On lave l'écheveau dans cette eau bouillante, puis plusieurs fois dans de l'eau claire, et on le fait sécher au soleil. La solution ne doit être ni salée ni trop fade, car une solution trop salée abîme la soie, et une trop fade ne la nettoie pas suffisamment : l'on ne doit pas laisser l'écheveau trop longtemps dans le bain, sinon cela abîmerait la soie. Comme préparer soi-même la potasse prend du temps, on l'achète souvent toute faite à la petite épicerie chinoise. Après le bain de potasse, on rince les écheveaux à l'eau claire.

Pour appliquer les teintures sur les fils, l'écheveau est passé autour d'un bâton et tourné dans la marmite de terre contenant l'eau et le colorant, pour qu'il prenne bien la couleur ; il faut $600 \mathrm{~g}$ de teinture pour 3 damloeung de soie (1 damloeung : $37,5 \mathrm{~g}$ ). La marmite est posée sur un feu pour maintenir le liquide à forte température, mais sans laisser bouillir. 
La durée du bain de teinture est de 15 à $20 \mathrm{mn}$ pour un meul de soie. L'écheveau est ensuite abondamment rincé à l'eau, frappé contre une planche propre pour que la couleur pénètre uniformément dans les fils, puis laissé sécher au soleil deux jours durant pour s'assurer que la couleur tient bien.

Pour que la teinture se fixe bien sur le fil, il a fallu aussi ajouter un mordant : un peu d'alun, sach chouv ( « chair acide »), qui est du sulfate double d'aluminium et de potasse, acheté au marché ( 15 à 20 g.). Ou bien, des feuilles et des jus de fruits très acides, mchou prey, «acides sauvages » : feuilles de tamarinier, daem ampeul, Tamarindus indica, jus de citron, fruits comestibles acides de daem trâlung thung, Averrhoa bilimbi, ou de daem sandân, Garcinia merguensis (Stoeckel 1921-1923 : 397, Martin 1971, Sek 1973 : 32). $600 \mathrm{~g}$ de teinture pour 3 damloeung de soie.

7 Après la teinture, la soie est plongée dans une nouvelle marmite d'eau bouillante, ce qui fixe la couleur. Après un bref repos $(5 \mathrm{mn})$, on plonge l'écheveau 5 à $10 \mathrm{mn}$ dans de l'eau froide ordinaire, pour éliminer les excès de teinture. Ou, souvent, on lave longuement les écheveaux dans de l'eau de pluie à froid, jusqu'à ce que l'eau de lavage reste claire.

Enfin, on amidonne l'écheveau en le faisant tremper dans une eau de riz bouillie, puis en laissant ensuite l'écheveau sécher au soleil. Cette opération rend le fil plus rigide; et le travail du tissage sera plus facile, car les fils vont moins s'emmêler. Au cours du tissage, la femme brossera régulièrement la chaîne, avec une brosse trempée dans le bain de riz bouilli ; la trame glissera mieux ainsi entre les fils de chaîne.

Les teintures végétales les plus pratiquées étaient le jaune, les différentes nuances de rouge, l'indigo et le noir.

\section{La couleur jaune}

10 La teinture jaune donnera de l'éclat aux couleurs, qui se fixeront mieux sur les fils. Aucune teinture ne se fera sans ce premier bain de teinture jaune.

11 1. La teinture obtenue de l'écorce (sambak en khmer, kdoh en cham) de l'arbre prahout ( Garcinia Vilersiana, Guttiferae) (Stoeckel 1921-1923 : 397, Martin 1971:68), donne une coloration jaune-safran, qui se conserve longtemps. D’ailleurs, plus on a prolongé le bain de teinture, meilleur il est. On trouve cet arbre dans de nombreuses régions du Cambodge. Quand on détache l'écorce de l'arbre, il faut bien veiller à ne pas en prendre en excès, ainsi l'écorce repoussera en un ou deux ans ; sinon, l'arbre mourra.

Pour obtenir le colorant, on hache les écorces en petits morceaux, puis on les pile dans un mortier. Les petits fragments d'écorces sont mis dans un sac et placés dans une marmite en terre cuite pleine d'eau, à quoi est ajouté un mordant, 30 ou $40 \mathrm{~g}$ d'alun, et des fruits acides, comme du citron, qui vont fixer la couleur. Si l'on en met trop, la couleur sera trop foncée ${ }^{2}$. On laisse bouillir pendant plusieurs heures, pour que l'eau s'évapore en grande partie, puis on filtre, pour ne garder que l'extrait jaune.

Pour être teinte, la soie en écheveau est placée dans une marmite contenant le mélange : colorant, alun fixateur et acides mordants. On place d'abord l'écheveau de soie dans le liquide froid ; puis l'écheveau est remué avec précaution dans l'eau portée à environ $90^{\circ} \mathrm{C}$, pendant un peu plus de $10 \mathrm{mn}$. On ajoute de l'alun si l'on juge que la teinte obtenue n'est pas assez marquée. Quand la soie a absorbé tout le colorant, on jette l'eau restante. L'opération est répétée deux ou trois fois, avec un nouveau bain de teinture et une pincée 
d'alun, jusqu'à ce que la teinte obtenue soit satisfaisante. Pour que la couleur soit bien uniforme, l'écheveau est battu une dizaine de fois sur une cuve de bois rectangulaire légèrement creusée avant d'être lavé à l'eau, puis séché.

Pour teindre un meul de fils de soie (2,5 niel de $600 \mathrm{~g}$, soit $1,5 \mathrm{~kg}$ ou $60 \mathrm{~m}$ de soie), il faut 6 meul de prahout Garcinia vilersiana, et $150 \mathrm{~g}$ d'alun. Ce grand arbre vient dans les forêts montagneuses de Kompong-Speu, et des provinces de Stung-Treng et de Kompong-Cham. L'écorce s'achetait au marché, où elle était facile à trouver, ou chez l'épicier chinois. La teinture au prahout est également connue et utilisée au Laos. Les fruits, à la fois sucrés et acides, peuvent être consommés.

Figure 4 : Garcinia vilersiana, Takeo, 1968

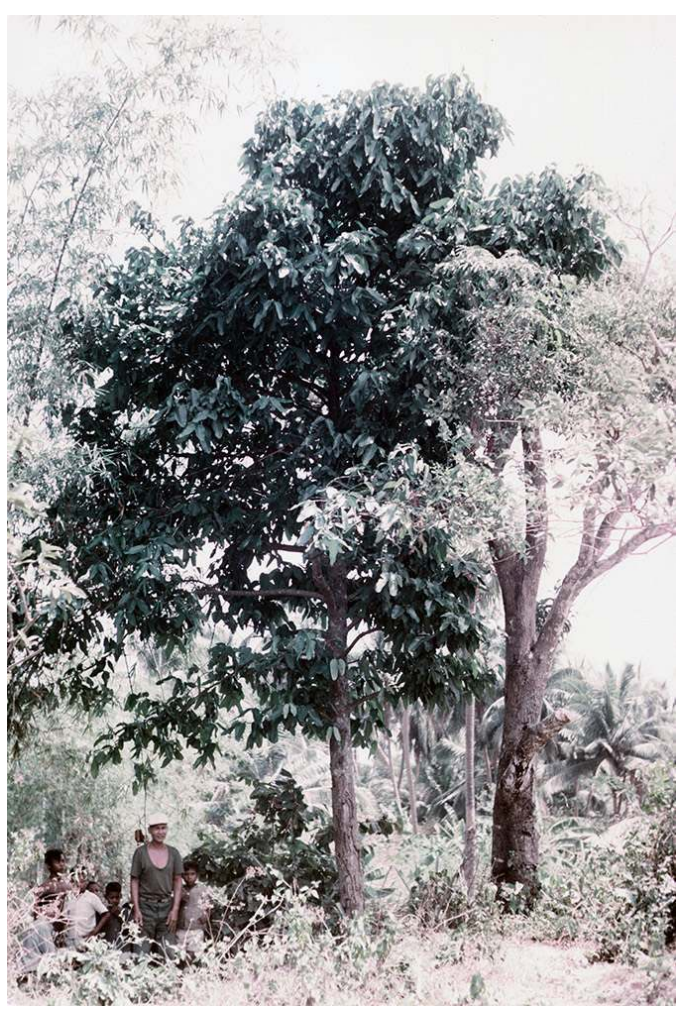

(C) B. Dupaigne tranches fines, séchés, réduits en poudre et bouillis pour donner une teinture jaune bistre qui sert à colorer les vêtements des bonzes (Teston \& Percheron 1931: 928, Stoeckel 1921-1923 : 397, Vidal et al. 1969, Martin $1971: 163$ ). On pouvait l'utiliser autrefois pour les écharpes, kramâr, de coton, mais la couleur ne tient pas bien. On ne l'emploie pas pour la soie. 
19 3. Le cœur (khlem) du tronc du "jacquier sauvage», daem khnao prey, donne une teinture jaune pour les vêtements des bonzes, comme en Birmanie, Thaïlande et Laos ${ }^{4}$. De petits morceaux du tronc d'un jacquier cultivé ou sauvage sont bouillis dans de l'eau. On filtre le liquide obtenu qui sera utilisé comme bain de teinture (pour le coton seulement), en y ajoutant un mordant (de l'alun) et des végétaux acides (Stoeckel 1921-1923: 397, Vidal et al. $1969: 209$, Morimoto $1995: 27)$. Cette teinture de jacquier donne un jaune orangé. À Prek Kdam, au nord de la capitale, on l'utilisait peu, car, à trop couper l'arbre, on n'obtient plus ses fruits, qui se vendent facilement, et cher.

4. Smâch chroluok. Des fragments d'écorces du tronc de Bruguiera gymnorrhiza sont hachés et placés en décoction dans une marmite d'eau chaude. La teinture se fait en ajoutant des mordants acides végétaux (Teston \& Percheron 1931 : 927, Sek 1973 : 26-27, Pavie 1884 : 56).

21 5. Feuilles de daem phlong, Memecylon edule (Melastomaceae) (Martin 1971:114, Stoeckel 1921-1923 : 397). Ce petit arbre, au tronc de la grosseur du poignet, de 2 ou $3 \mathrm{~m}$ de haut, se trouve principalement dans la région de Kompong-Cham. Les feuilles sont préalablement séchées au soleil ; on les fait bouillir dans une marmite, une poignée de feuilles pour une marmite de 5 ou 101 d'eau. On rajoute des feuilles acides de sandân (Garcinia merguensis), et l'on filtre la solution. On obtient un jaune clair qui permet de teindre des fils de soie. En ajoutant ce colorant jaune à la solution de la teinture rouge, leak khmer, on obtient une belle couleur rouge vif.

22 Si on n'en met pas dans cette teinture rouge, la teinte obtenue sera considérée comme trop foncée.

23 Si on n'a pas de feuille de sandân, on peut utiliser les feuilles acidulées de la liane thnong , Aganonerion polymorphum, que l'on trouve facilement sur les marchés (Martin 1971 : 131).

24 À Prek Kdam, les Malais, dont les sarongs sont renommés, employaient cette teinture depuis longtemps. Une brassée de feuilles se garde un ou deux ans. On en achetait deux ou trois brassées pour l'année, à des Khmers, qui allaient les chercher près des collines.

6. Le cœur de la liane épineuse khlé(a), abondante dans la région de Kompong Cham et dans les régions inondées autour du Tonlé Sap, donne une teinture jaune (Aymonier 1885, Bouinais \& Paulus 1885 : 534, Teston \& Percheron 1931 : 927, Stoeckel 1921-1923 : 397). On la casse en morceaux, que l'on fait bouillir. On filtre la solution. Il faut trois bains de teinture, avec une heure trente de cuisson à chaque fois, dans un bain qui contient également du colorant jaune prahout et un mordant, l'alun. Cela donne une couleur jaune orangé. Utilisée seule, sans être relevée de prahout, on obtient une couleur terne et trop foncée. On l'utilisait pour les jupes phamuong et kaniuh des femmes.

Chaque année, des colporteurs khmers venaient en proposer. On en employait beaucoup autrefois, moins maintenant, et les gens ne vont plus en chercher dans les mares ${ }^{5}$.

7. L'écorce de daem khtum, Nauclea brunosis, abondant dans les forêts inondées, est citée comme teinture et drogue médicale (Stoeckel 1921-1923 : 398, Martin 1971 : 123). À Prek Kdam, on la connaît anciennement comme un médicament, et non comme teinture. Après son accouchement, si la femme éprouve des difficultés à se nourrir, on mélange l'écorce à d'autres ingrédients, du crang sekrey (NS), de l'ail blanc, khtoeum sâ, coupé en sept, avec sept grains de poivre, mrec (Piper nigrum) (Martin 1971:148 et 169). On pile le tout, on met dans de l'eau en ébullition et le breuvage est bu quand il est tiède. 
L'écorce du kdol, arbre très répandu, citée comme produit tinctorial (Stoeckel 1921-1923 : 398), est connue à Prek Kdam comme médicament contre la fièvre ${ }^{6}$.

Les écorces et les racines de sramar (NS), sont citées comme teintures ${ }^{7}$. La plante est également connue à Prek Kdam comme un médicament : les graines sont écrasées sur une pierre creuse ; on mélange la pâte obtenue avec de l'eau, et on la met dans l'oreille pour calmer les douleurs.

Figure 5 : Renvidoir de bois sculpté Khmer. Préparation des fils à la teinture, village de Prek Chan Krân, province de Prey Veng, 1969

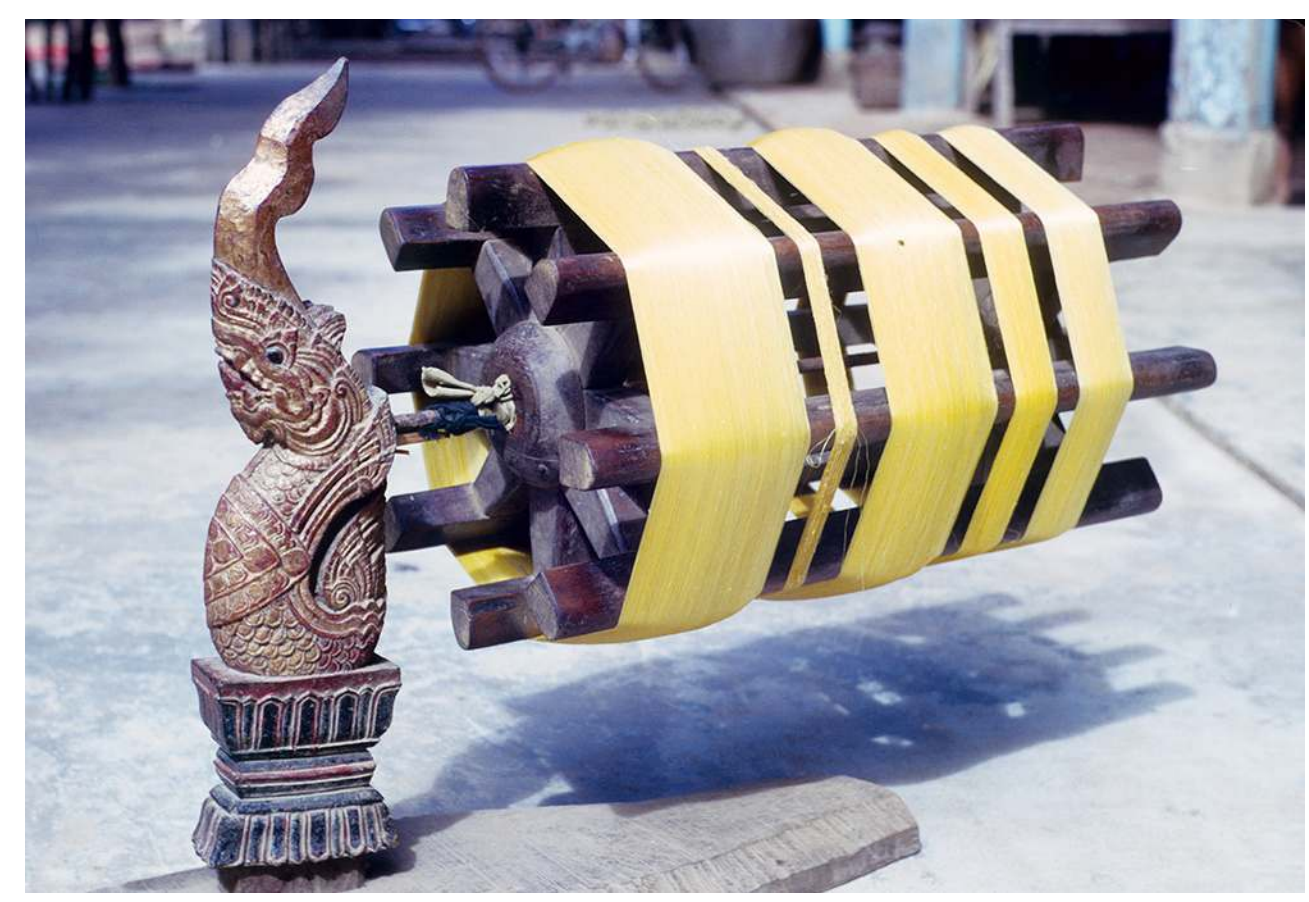

(C) B. Dupaigne

\section{Le rouge, krahom}

Cette couleur est fournie principalement par le stick-lac.

31 1. Le stick-lac, leak khmer, est d'origine animale. On le trouve facilement en Inde, en Birmanie, en Thaillande, au Cambodge ${ }^{8}$ et surtout au Laos. C'est une teinture qui a bien résisté à la concurrence des produits chimiques, et qui donnait lieu à un important commerce. Elle s'achetait chez l'épicier chinois; le résidu pâteux de teinture, ach, est utilisé comme colle forte par les menuisiers, pour fixer les manches d'outils, les socs de charrue, l'assemblage de meubles, ou pour réparer une poterie brisée ; mais, à cause de sa couleur rouge, on ne l'utilise pas pour les meubles. On employait également les résidus de la teinture "en médecine en emplâtre pour les individus qui ont fait des chutes " (Bouinais \& Paulus 1885). Le docteur Harmand remarque en 1875 l'importance de son commerce dans la région de Kratié (Harmand 1876 : 341-342).

La cire à cacheter était faite avec cette laque, "produite en grande quantité par le Cambodge, mais qui va aussi en majeure partie alimenter le commerce de Singapore " (Kloss 1896). Sous le Protectorat français, existait une usine de transformation de cette gomme-lacque à $125 \mathrm{~km}$ de Hanoï ${ }^{9}$. Elle était exportée pour confectionner des vernis, et 
autres objets semi-rigides. Pendant quelque temps, «le stick-lac a été particulièrement employé pour les disques de phonographes, les isolants électriques, les vernis et la fabrication de pâte à joints hermétiques $»^{10}$.

Il ne s'agit ni d'une gomme ni d'une laque, mais d'une résine provoquée par les piqûres de l'insecte kra (ou mei), un hémiptère (ou rhynchote), Kcoccuserria lacca, (syn. Laccifer lacca) Laccifer lacca (dit aussi Kerria lacca, Tachardia lacca, Carteria lacca, Coccus cacti coccinellifera), qui vit en colonies parasites sur les écorces de branches de différents arbres forestiers ou cultivés ${ }^{11}$. Les plus appréciés sont :

Combretum quadrangulare, sangkae, dont les feuilles servent aussi à enrouler le tabac à fumer ; il est cultivé autour du lac de Tonlé Bati (Martin $1971: 108)^{12}$.

Le latanier Corypha Lecomtei, daem treang, des Palmaceae.

Dalbergia nigrescens, daem snuol, des Fabaceae.

Sindora cochinchinensis, daem krokoh, des Fabaceae (Martin 1971 : 99).

Ficus benjamina, daem chrey krem, F. religiosa, daem po (Brenier 1930:174) ${ }^{13}$, des Moraceae.

Puis, viennent le «jujubier indien », Zizyphus jujuba, daem putria, des Rhamnaceae (Martin 1971: 87).

Shorea Cochinchinensis, daem propél msau, des Dipterocarpaceae (Sek 1973 : 28)

Schleichera triguda, daem pongrô, des Sapindaceae (Martin 1971 : 91)

Butea frondosa, des Fabaceae.

L'élevage de ces insectes pour la production de la laque rouge est très ancien en Asie orientale. É. Aymonier nous en parle en 1900 pour la commune (srok) de Bati (province de Takeo), très impliqué dans les divers tissages de soie :

«Ces habitants savent distinguer et trier les beaux insectes à laque pour la reproduction, les attachant par petits sacs, en juin, aux arbres chréi (un figuier), sangkê et treang, n'oubliant pas d'invoquer au préalable les génies protecteurs, allumant bougies et baguettes odoriférantes près d'un bel arbre et demandant que ces insectes soient préservés des maladies et des mandibules des fourmis dévorantes » (Aymonier $1900: 173)$.

«Les jeunes larves se fixent sur les rameaux nouveaux, transformant la sève qu'elles pompent en une excrétion (cire, résine et colorant) les entourant. Elles se ménagent des alvéoles où elles évoluent. Les mâles sortent en premier pour féconder les femelles qui restent dans leur logement jusqu'à maturité des œufs, avant de se fixer sur d'autres branchages. La couche d'exsudat atteint de 5 à 12 $\mathrm{mm} »$ (Teston \& Percheron 1931 : 779, 906 et 944 ).

Les insectes sont installés au début de la saison des pluies (aux mois de Bos et Mir) sur les branches d'un arbre dont ils suceront la sève, et où ils formeront des essaims exploitables au bout d'une année. Les œufs des insectes éclosent en petits vers, me', que l'on replace sur les branches de l'arbre. On fixe avec une ficelle les essaims sur des branches, ou bien on y accroche des tissus contenant un essaim. Les larves, srâmâl, toutes petites et rouges, en sortent et, au bout de quatre ou cinq jours, commencent à s'enfermer dans leur nouvel essaim, appelé ach leak (« excréments de laque»). Il faut protéger l'arbre contre les fourmis, ângkrâng, en badigeonnant le tronc de chaux: ces fourmis «avec un liquide dans l'estomac qui brûle comme de l'alcool ", attaquent les larves pour s'en nourrir. La récolte se fait de mars à mai; on coupe les branches qui portent les essaims, elles 
repousseront à la saison des pluies. Si on a obtenu une bonne récolte, on vend les excédents au commerçant chinois, plus ou moins cher selon la période.

Les essaims destinés à la vente ou à l'usage domestique sont exposés au soleil pour tuer les insectes, qui donnent le stick-lac. Pour la reproduction, on garde des morceaux de branches que l'on conserve à l'abri du soleil durant toute la saison chaude, et que l'on attache sur les branches de l'arbre au début de la saison des pluies. Il faut bien s'appliquer à ne pas laisser les exsudats à proximité des trous entre les poteaux des maisons, car les insectes s'y cacheraient.

Cette culture est considérée comme facile : il n'y a pas d'autre travail que d'accrocher et de récolter. Cependant cette culture, tout comme la teinture, est l'objet d'interdits. En effet, le processus d'obtention obligeant à tuer les insectes, certaines paysannes hésitent à le pratiquer : «cultiver la gomme-laque, capturer des éléphants, brûler la forêt, élever des vers à soie, toutes ces actions vont contre l'interdiction de Bouddha de tuer des êtres vivants $»^{14}$. La teinture, qui se fait avec des êtres vivants, les insectes, ne peut également réussir que si l'on respecte les interdits. Le principal, selon les teinturières, est que « Les femmes enceintes, ou qui sont en période de règles, ne doivent pas approcher le bain de teinture, sinon la couleur ne prendrait pas. Pour protéger la teinture de tout regard nuisible, on travaille dans une pièce bien close »: le sang ne doit pas approcher la teinture en train de se faire ${ }^{15}$.

Ces femmes ne peuvent approcher le travail de teinture en cours, ni se tenir près de la teinturière : le contact avec le sang est dangereux pour les actions en cours. Sek You Oun ajoute qu'au moment de la mort de celui qui a élevé ces insectes, les essaims tombent de l'arbre. De même, les cercueils ne doivent pas passer à proximité des arbres abritant les insectes, sinon ils mourront (Sek 1973: 30). Au Laos, où le stick-lac reste un produit d'exportation, É. Aymonier notait aussi l'interdit que « les gens qui s'en occupent doivent s'abstenir de se laver la tête, sinon les insectes à laque prendraient mal » (1885:40).

La teinture se prépare en broyant le stick-lac dans un mortier avec de l'eau froide que l'on remue sans cesse ; on laisse reposer pendant un à deux jours, afin que le principe colorant sorte. Puis, le mélange est soigneusement filtré à travers une natte pour obtenir le bain de teinture en éliminant la résine et les restes végétaux. On fait chauffer une demi-heure environ le mélange dans une marmite d'eau bouillante gardée sur le foyer de feu de bois. Une deuxième, puis une troisième cuisson donneront une teinture moins diluée ${ }^{16}$.

49 L'écheveau de fils de soie, toujours teints préalablement en jaune au prahout, est plongé à froid dans un bain d'eau additionnée d'un mordant, de l'alun chimique, sach chouv, et des feuilles et des fruits de tamarinier acide; puis il est essoré avant la teinture ${ }^{17}$. La teinture avec ce « carmin de cochenille » se fait ensuite à chaud. On rajoute de l'alun si la couleur n'est pas assez marquée, et on recommence le bain bouillant plusieurs fois, jusqu'à ce que la couleur soit jugée satisfaisante (on peut aller jusqu'à quinze fois). Enfin, on lave l'écheveau à l'eau froide, on l'essore. La teinturière se lave alors les mains avec de l'eau additionnée d'un fruit acidulé. Le bain de colorant ne servira que pour la teinture d'un seul écheveau. Pour les prochains, il faudra préparer un nouveau bain de teinture, avec un colorant frais.

50 À Krokar, dans la province de Kompong Cham, comme à Prek Chang Krân en Prey Veng, les feuilles acides utilisées sont le sandân, qui donnera une couleur rouge ou orange, tum . La feuille de la liane commune, slek thnong donnera du rouge œuf de fourmi rouge comestible (pong angkrâng). Les feuilles du tamarinier slek âmpeul, (Tamarindus indica) 
donneront un autre rouge (Martin 1971: 67, 99 et 131). Un ajout de jus de citron, kraûch chhma, donnera un rouge clair, semblable à la teinte du lotus. Cette teinture est encore utilisée en Bati et Samrong (Takeo) et à Angkor Chey en Kampot (Morimoto 1995 : 25). (Leguminosae), arbre qui peut atteindre 10 mètres de haut, fournissent un glucoside employé pour des teintures roses, rouges ou violette, pour les nattes de jonc ${ }^{20}$. Pour obtenir la couleur rouge, on fait macérer dans une marmite d'eau bouillante du cœur, khleam, de bois de sbaeng, des graines de fruit du chompu (à la fois rocouyer et jambosier), et un peu d'écorce de smac (Martin 1971: 112) ${ }^{21}$. La solution obtenue est mélangée avec du jaune de curcuma pour obtenir un rouge écarlate. Pour le rouge foncé, on n'ajoutait pas de curcuma ${ }^{22}$. Le mordant vient de végétaux acides. A. Pavie parle en 1884 du commerce de ce bois de sappan en Kandal, dans la commune de Kandal Stung (Pavie $1884: 6$, Aymonier $1880: 367$ ). pierre, on en met la couleur sur le front des bébés pour leur éviter les maux de tête. On peut aussi en préparer un médicament "pour traiter les maladies de la peau ( (Martin 1971 : 96). desséchées du Carthamus tinctorius, Compositae. Les fleurs sont mises à bouillir, avant que le liquide ne soit tamisé.

«Ces fleurs renferment deux matières colorantes, l'une jaune, l'autre rouge. On élimine la couleur jaune en lavant les fleurs à l'eau acidulée. Les fleurs ainsi lavées sont traitées par une solution de carbonate de soude. Ce carthame peut être utilisé pour la soie et rarement pour le $\operatorname{coton}^{23} \%$. 
La racine de la garance des teinturiers, Rubia tinctorum, si connue en Asie occidentale, ne semble pas être utilisée au Cambodge.

Figure 6 : Bains de teinture rouge, Prek Chang Dek, province de Prey-Veng, 1969

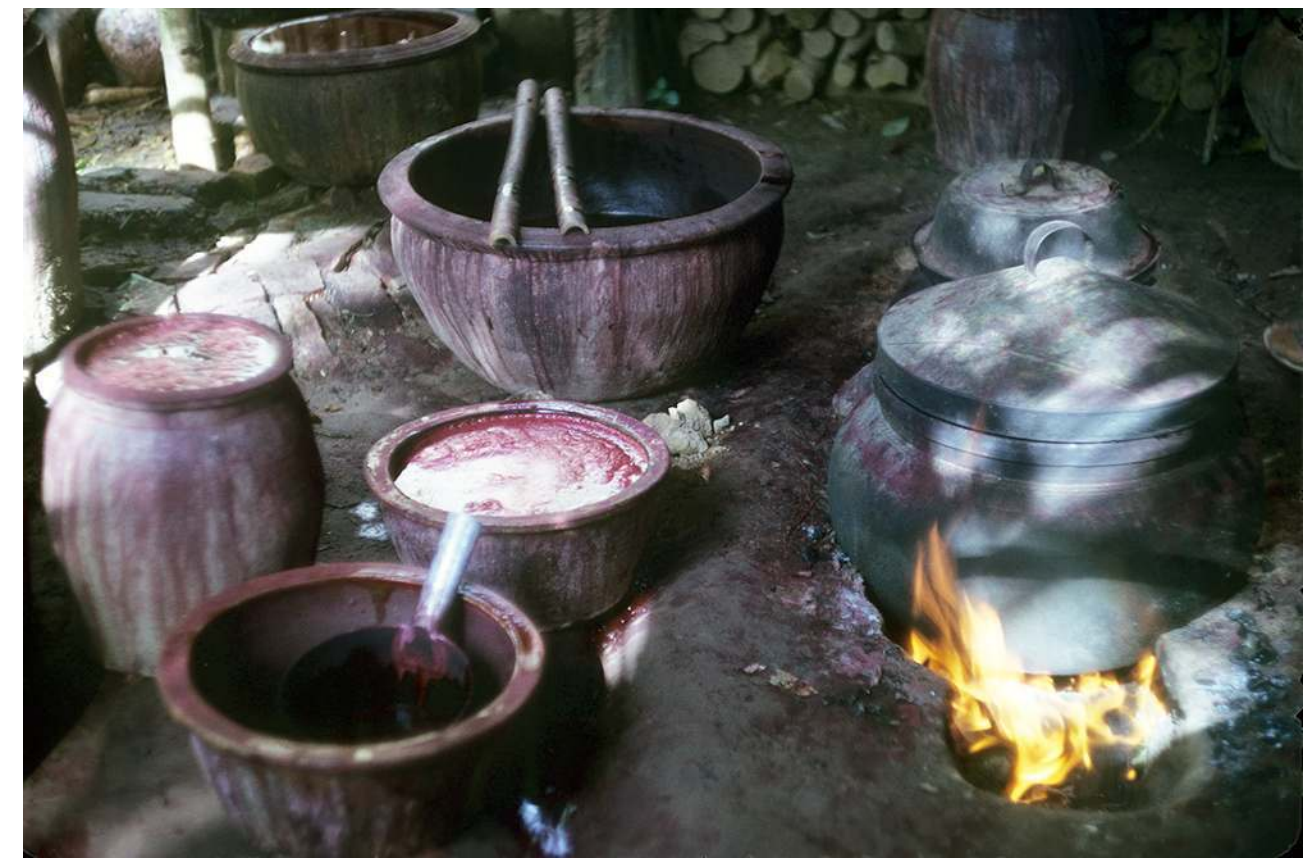

(C) B. Dupaigne

Figure 7 : Teinture de l'écheveau en rouge, Srok de Bati, Takeo, 1968

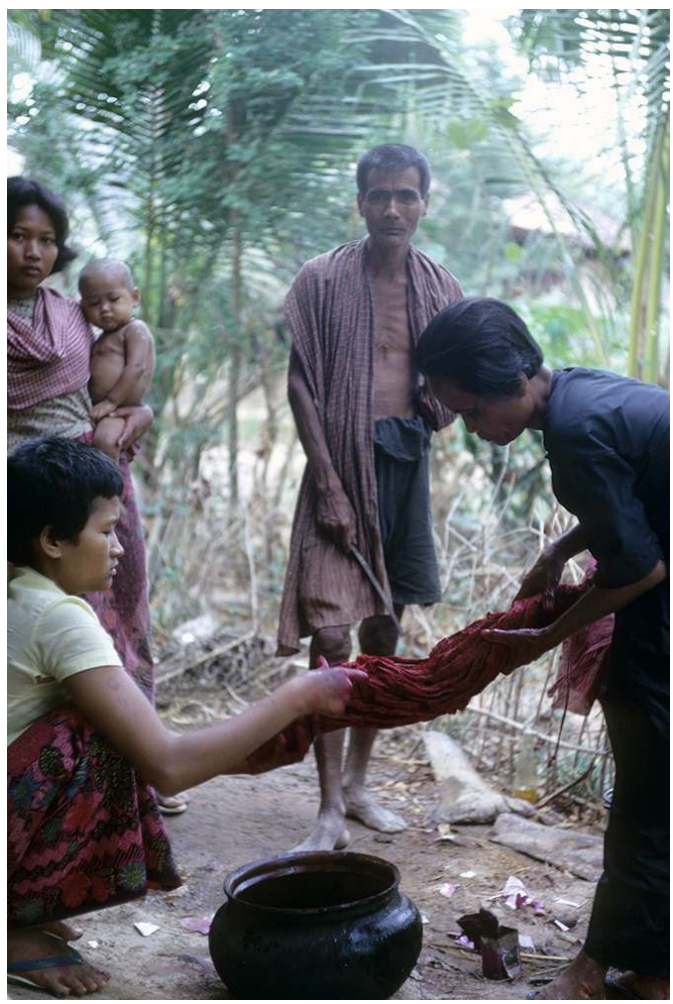

(C) B. Dupaigne 
Figure 8 : Teinture de l'écheveau en rouge, Prek Chang Krân, province de Prey-Veng, 2011

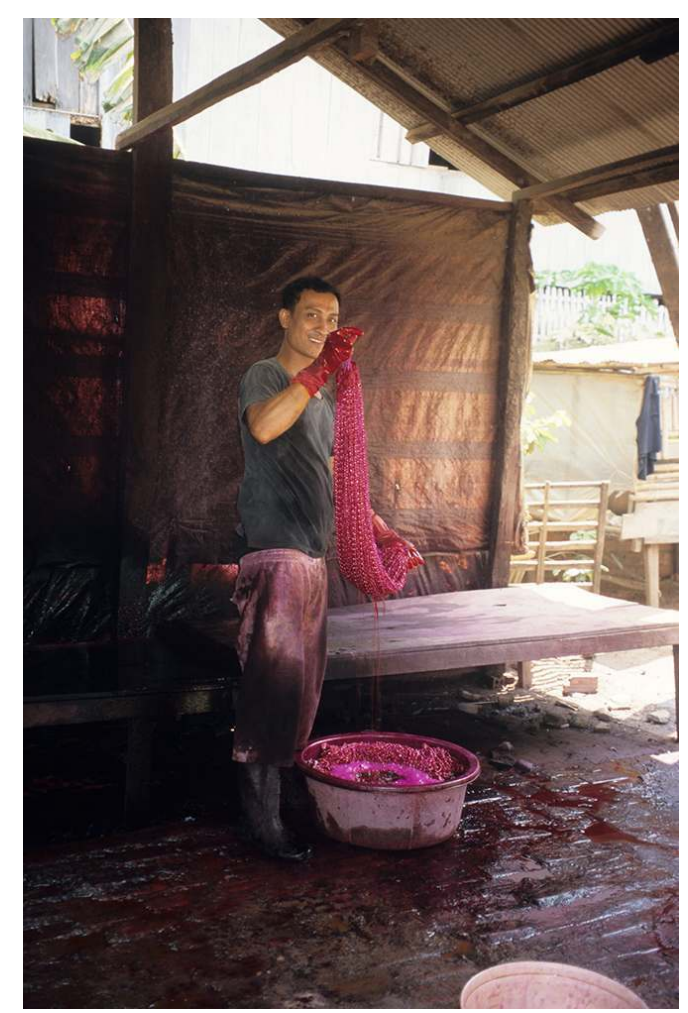

(c) B. Dupaigne

\section{L'indigo}

Cette couleur est obtenue par fermentation des feuilles et des tiges de l'indigotier, daem trôm, Indigofera tinctoria, fabacée qui était cultivée depuis des temps immémoriaux à grande échelle sur les berges et dans les îles du Mékong, près de Kompong-Cham, ainsi que dans le delta du Mékong au Vietnam, en Inde et en Asie du Sud-est. L'indigotier demande un terrain riche, c'est pourquoi on en sème les graines surtout en décembre ou janvier (Méakh) sur des terres de berge, meubles et humides, des terres d'alluvion inondées chaque année par les crues du Mékong, lors de la saison des pluies. La plante a également un usage médicinal : à Koh Sautin, elle intervient en onguent contre le goître, fait à partir d'une eau dans laquelle a trempé toute la plante.

Un industriel français du Cambodge, M. Caraman, à la « Ferme des Mares », avait fondé de grands espoirs en 1882 sur sa culture au niveau industriel quand la teinture était réclamée par les usines textiles françaises. Il obtenait une pâte à 45 à $50 \%$ d'indigotine, quand la moyenne au Bengale était de 40 à $50 \%$, et la vendait facilement deux fois son prix de revient à Londres, Hambourg, Marseille, Bordeaux, Le Havre ${ }^{24}$. Mais la teinture à l'indigo a été concurrencée par les teintures chimiques, d'emploi plus aisé : à partir de 1878, des chimistes allemands synthétisent l'indigo. La firme Baeyer produit dès 1882 un indigo commercial, qui s'est répandu progressivement à travers le monde pour sa simplicité d'usage.

61 En 1904, l'indigo occupait 7000 hectares de terres de berge au Cambodge. Les agriculteurs livraient le colorant 
« en pâte plus ou moins liquide, suivant qu'il a été plus ou moins égoutté. Cette pâte, de couleur bleu-noir, ne contient, d'après les analyses, que 2,5 à 2,75\% d'indigotine ; le reste représente de la chaux, des matières minérales et de l'eau. Des essais récents, soit du traitement de la plante par des procédés nouveaux, soit de l'extraction de l'indigotine de l'indigo indigène, ont donné de bons résultats. Malheureusement, les cultures sont très disséminées ${ }^{25}$. une jarre et l'on rajoute de l'eau pour la remplir à ras bord. Pour que la teinture prenne bien, on ajoute au bain du jus de citron, quelquefois du gingembre pilé, des cendres filtrées de kapokier (donc de la potasse), des coquillages réduits en cendres (donc de la chaux), des morceaux de bananes et du sucre de palme ou de canne à sucre qui améliore la teinture ${ }^{33}$. 
69 peut plonger sept à dix écheveaux à la fois. L'on place l'écheveau dans le bain, toujours à
froid. La teinte obtenue est plus ou moins foncée, selon le temps que l'on laisse l'écheveau
dans le bain et le nombre des bains successifs. au soleil, puis on pratique un deuxième bain, ou un troisième bain, de la même durée. On voit la couleur apparaître, d'abord vert clair, puis plus foncé, puis bleu clair et finalement plus foncé. L'écheveau doit être séché au soleil, et l'oxygène lui fera prendre sa couleur indigo. Pour teindre d'autres écheveaux, il faut utiliser une nouvelle solution de pâte d'indigo, sinon le bleu obtenu sera trop clair. La teinture indigo sur un fil de soie blanche donne une teinte bleue ; sur un fil jaune, elle donne du vert foncé ; sur un écheveau déjà teint en rouge, du violet, presque noir ${ }^{34}$. En fin d'opération, on rince longuement l'écheveau dans l'eau du fleuve, pour évacuer l'excédent de teinture.

Figure 9 : Culture d'indigotier, Koh Sautin, province de Kompong-Cham, 1969

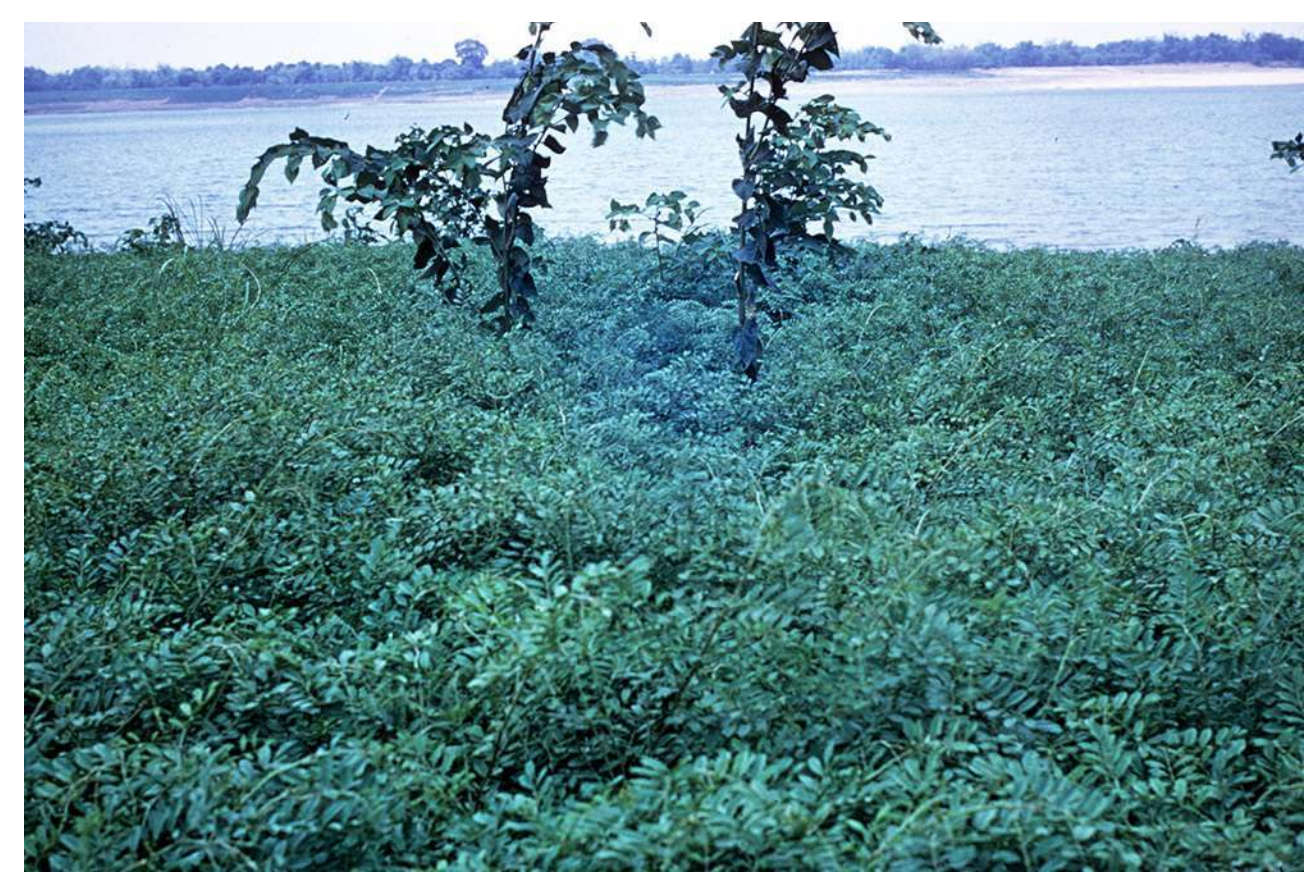

(c) B. Dupaigne

\section{La couleur verte}

71 Elle ne s'obtient pas directement. Les écheveaux plongés à froid dans la teinture indigo apparaissent d'abord vert, avant de passer au bleu profond au fur et à mesure des bains de couleurs. Ceci, d'autant plus que l'écheveau aura été préalablement teint en jaune avec le prahout. Le passage à la teinture jaune, puis à l'indigo, donnera un vert de bonne qualité, plus ou moins foncé.

72 Un écheveau déjà teint en jaune à l'écorce de manguier, Mangifera indica, svay, et plongé dans des bains d'indigo apparaîtra également vert (Dy Phon $2000: 427)^{35}$. 


\section{Le noir, khmau, thleas} provinces de Kandal et de Kompong-Speu. Les fruits, jaune-vert et ronds, utilisés comme source du colorant, mûrissent de mai à juillet, et sont cueillis encore verts. On dit que cet arbre a été introduit par des Siamois, et, en effet, son nom est d'origine thaï (Martin 1971 : 129, Girard $1887:$ 23-24, Blandin $1910: 604$ ). L'espèce makloeua kaèk (« corbeau »), dont le rendement en fruit est plus faible que le makloeua plouk (« ivoire »), donne des fruits plus riches en matières colorantes et plus recherchés des teinturiers ${ }^{36}$. Cette teinture est utilisée aussi bien pour les tissus de coton et de soie, mais elle ne convient pas pour la teinture des fils en écheveaux.

Le makloeua était planté surtout dans la province de Kandal (environs de Phnom-Penh, commune de Kandal Stung, de Khsach Kandal et de Muk Kompul), dans les communes Kong Pisei et Samrong Thong et Phnom Sruoch en Kompong-Speu, ainsi que dans l'île de Koh Sautin (Delvert 1961: 384). Les centres de teinture étaient ou bien des centres urbains (Takhmau et Banam), où des paysans pauvres étaient employés par des commerçants chinois, ou bien des marchés ruraux de la province de Kandal: Preak Tameak et Prek Prasap (srok de Khsach Kandal), Prek Koy (srok de Saang). Le travail s'effectue en ateliers d'une dizaine de personnes (hommes et femmes), les jarres étant enterrées à mi-hauteur sous un appentis.

«La récolte est faite soit par les paysans-propriétaires soit par des commerçants chinois qui achètent les fruits sur l'arbre. Les fruits sont utilisables pendant quatre ou cinq jours après la cueillette ; ils sont broyés au pilon à main dans un mortier, puis mis à tremper. Les tissus sont en général teints à l'indigo, puis trempés dans une solution de soude qui sert de mordant (eau mélangée de cendres de gousses de kapokier, de cendres de coquillages); ils sont enfin trempés 4 ou 5 fois dans l'eau de mackloeua contenue dans des jarres; battus, essorés, mis à sécher au soleil pendant quinze jours. On évite donc de teindre par temps incertain » (Delvert 1961: $146)^{37}$.

À Prek Kdâm, les Chams teignaient encore des étoffes ainsi, y compris celles en soie. Il fallait trois ou quatre jours de teintures successives, puis les étoffes étaient exposées au soleil. On achetait les fruits à des Khmers, et ces teintures n'étaient pratiquées que pour l'usage personnel.

Les tisserandes cambodgiennes obtiennent le noir en teignant les écheveaux de soie, successivement en jaune, rouge, puis indigo. « On n'utilise pas le makloeua pour teindre les écheveaux, car il faudrait trop les malaxer dans le bain de teinture, et les fils s'emmêleraient ", dit une tisserande de Takeo.

Dans la province de Kandal, à $37 \mathrm{~km}$ au sud de Phnom-Penh, des Vietnamiens perpétuaient une tradition de tissages de soie façonnée, à plusieurs rangs de lisses supplémentaires pour les dessins ${ }^{38}$. Les tissus, qui servaient surtout à confectionner les pantalons des Chinoises et des Vietnamiennes, étaient teints en noir par une série de bains, à froid, dans une solution décantée et filtrée des fruits broyés et macérés. Le mordant était fait de cendres de gousses de kapok ou de coquillages. Entre chaque bain, il fallait laisser sécher les tissus au soleil. En un jour, on pratiquait six à sept bains de 
teinture, jusqu'à dix s'il faisait très chaud. Outre la soie, cette teinture servait également pour des vêtements de coton d'usage.

Pour obtenir une jolie teinte noire, il fallait quinze jours de teintures, soit environ cent bains en tout. Les bains devaient être renouvelés périodiquement avec de nouveaux fruits pilés. La couleur tient alors très bien ; les jupes dont la teinte avait passé au soleil étaient reteintes. Il fallait $30 \mathrm{~kg}$ de fruits pour teindre $15 \mathrm{~m}$ de tissu de soie. Les arbres étaient nombreux dans cette province de Kandal, et leurs propriétaires qui ne pratiquaient pas le tissage et la teinture vendaient aux voisins les fruits de leurs arbres. Certaines tisserandes portaient leur production à Phnom-Penh pour être vendue au grand marché central.

Figure 10 : Tissu façonné teint au makloeua, Srok de Saang, province de Kandal, 1970

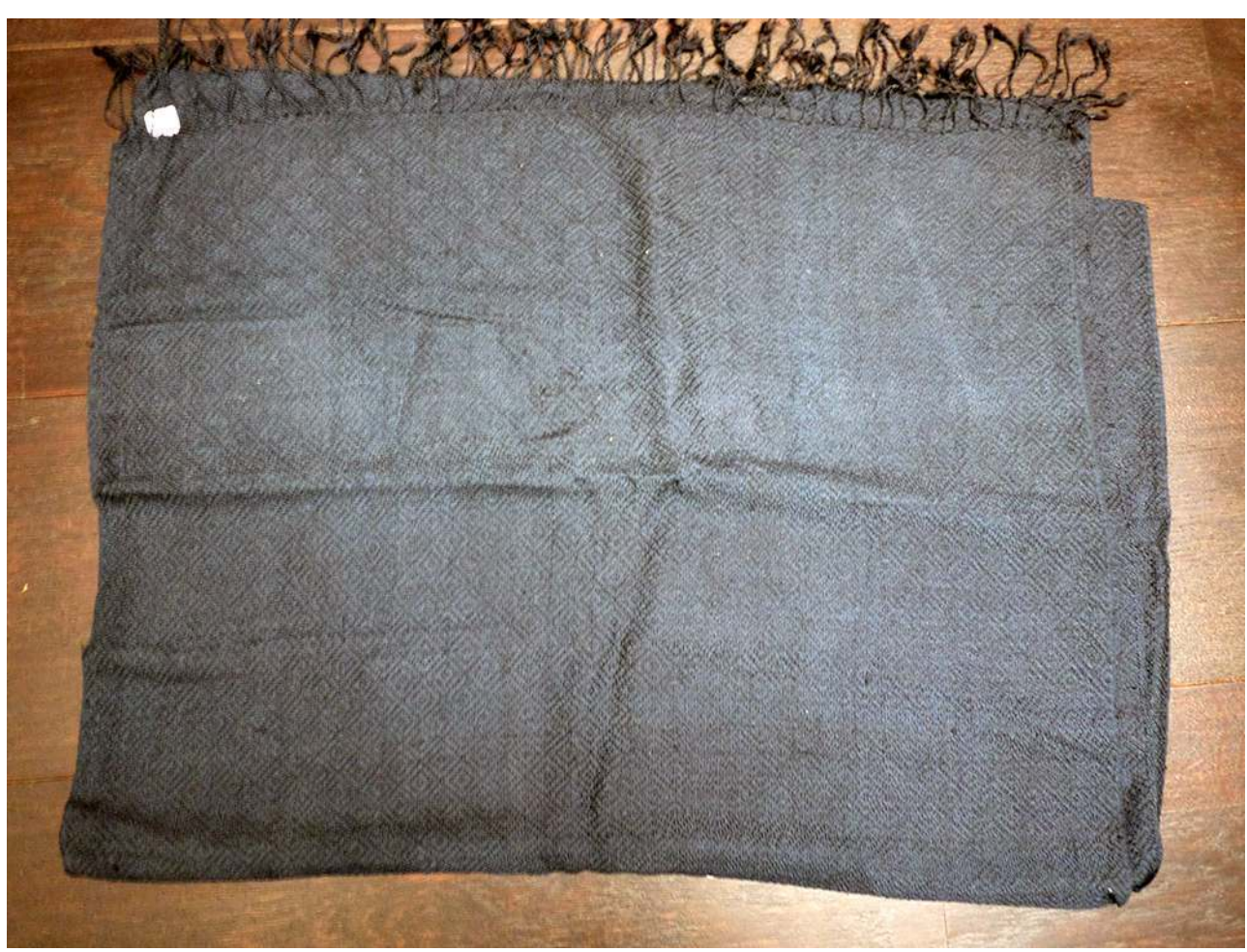

(c) B. Dupaigne

D'autres plantes pouvaient être utilisées pour la teinture en noir. Les feuilles et gousses de l'acacia, daem sombueor (Fabaceae), écrasées et additionnées de sulfate de fer, donnaient la couleur noire, après plusieurs bains successifs à chaud (Martin 1971: 94, Stoekel 1921-1923 : 398, Sek $1973: 24$ ). La plante avait également un usage médicinal.

81 Les feuilles du trobaek prey, "goyavier sauvage », Lagerstroemia floribunda, accompagnée de sulfate de fer donnaient également une teinture noire (Martin 1971 : 101).

«On pouvait aussi prendre de l'écorce de daem snuol (le Dalbergia nigrescens, famille des Papilionaceae (Martin 1971: 101)) à laquelle on ajoute de l'écorce du manguier sauvage, acide, et des daem phnum phneng, un fruit acide (Hymenocardia Wallichii, une Euphorbiaceae (Martin 1971: 157)) que l'on faisait macérer dans une grande jarre, ou bouillir dans une grande marmite. La solution obtenue donnait la couleur noire ${ }^{39}$ ».

82 La résine, moreak, de l'«arbre à laque », Melanorrhoea laccifera, provenant de l'arbre daem krêl, mélangée à de l'eau ne sert pas pour teindre les tissus, mais comme vernis pour les barques, les objets en fer et en bois (Stoekel 1921-1923 : 398, Martin 1971 : 93). 


\section{D'autres teintures}

Les Cambodgiens sont tous pêcheurs, plus ou moins, utilisant des filets de coton, aujourd'hui souvent de nylon. Pour leur entretien et les rendre moins perméables à l'eau, ils les teignent dans une décoction d'écorces de palétuvier sme, kon kang qui imprègne les fils de tanin protecteur ${ }^{40}$. Au Vietnam, cette teinture brune des filets est donnée par les tubercules de Dioscorea alata, cu nau, très tannifère également et bon protecteur contre le sel de la mer.

Figure 11 : Carte postale, Saïgon, vers 1930

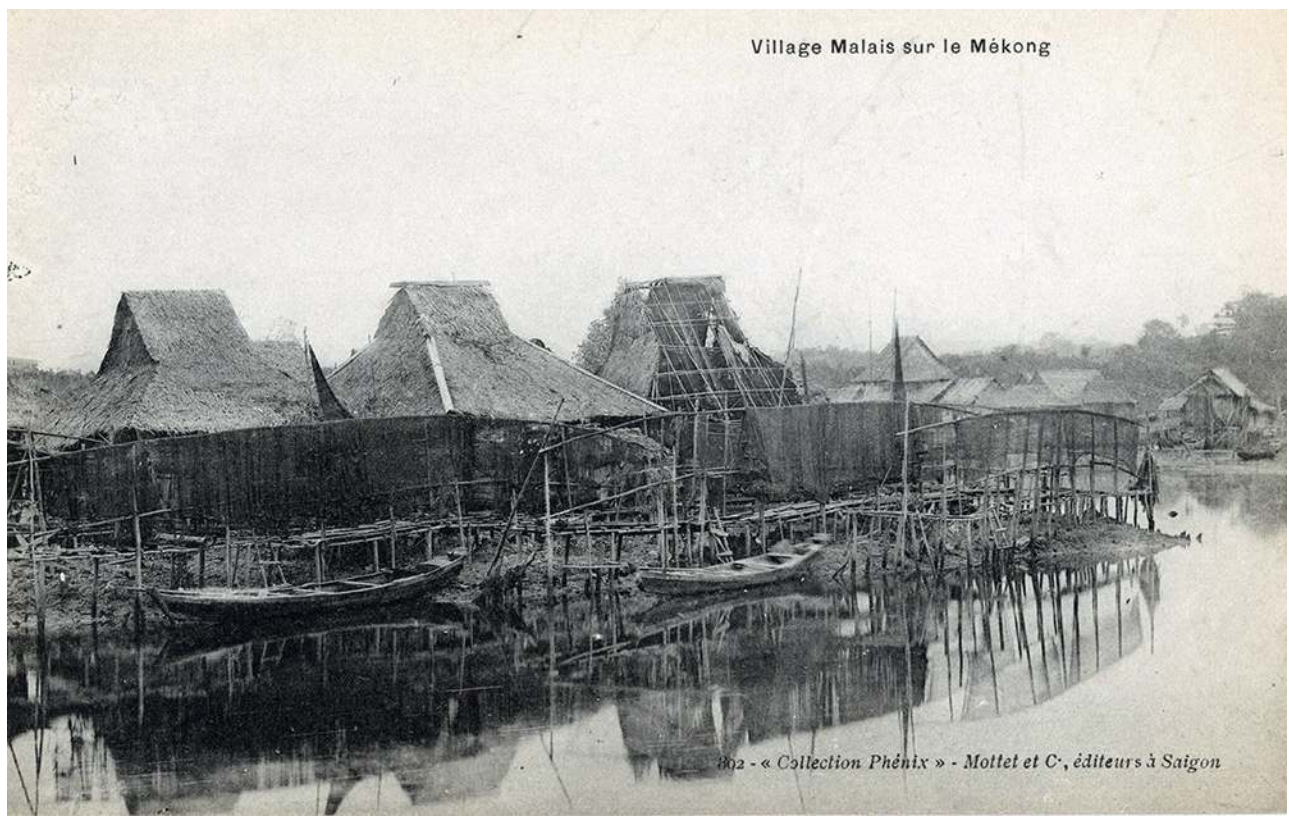

M. K. Morimoto (de l'Institute for Khmer Traditional Textiles) utilise également, comme teintures, pour le jaune, les feuilles de bougainvilliers (Bougainvillea glabra) et de bananiers, Musa spp., pour le rose et le brun, les écorces de noix de coco, Cocos nucifera, pour le gris et le noir, le bois de lychee, Lichi chinensis, pour le noir, les feuilles d'amandier, Terminalia catappa, un arbre américain (Aubaile-Sallenave 1987). 
Figure 12 : Teinturière de l'ethnie Cham, village de Chraing Chamrés, province de Kandal, 1968

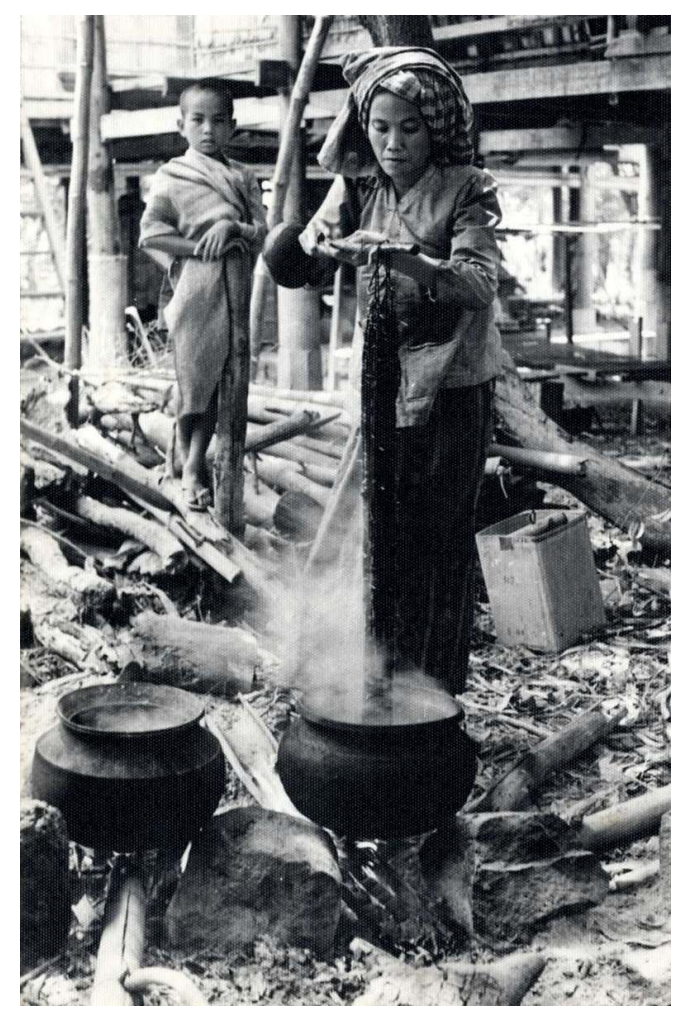

(C) B. Dupaigne

\section{Conclusion}

Tous ces procédés de teinture sont bien connus. Les tisserandes locales les négligent maintenant. Elles préfèrent teindre avec des colorants chimiques, importés du Vietnam, plus faciles d'emploi. Ou encore acheter directement des fils de soie déjà teints et prêts à l'emploi, produits également au Vietnam. Il doit rester des centres d'élevage des vers à soie, car, sur les marchés, sont encore vendues des chrysalides, considérées comme un délice. À moins que les vers ne soient plus élevés que pour en manger les chrysalides. 
Figure 13 : Vitrine du Musée de l'Homme. Cuve de dévidage des cocons de vers à soie, 1982

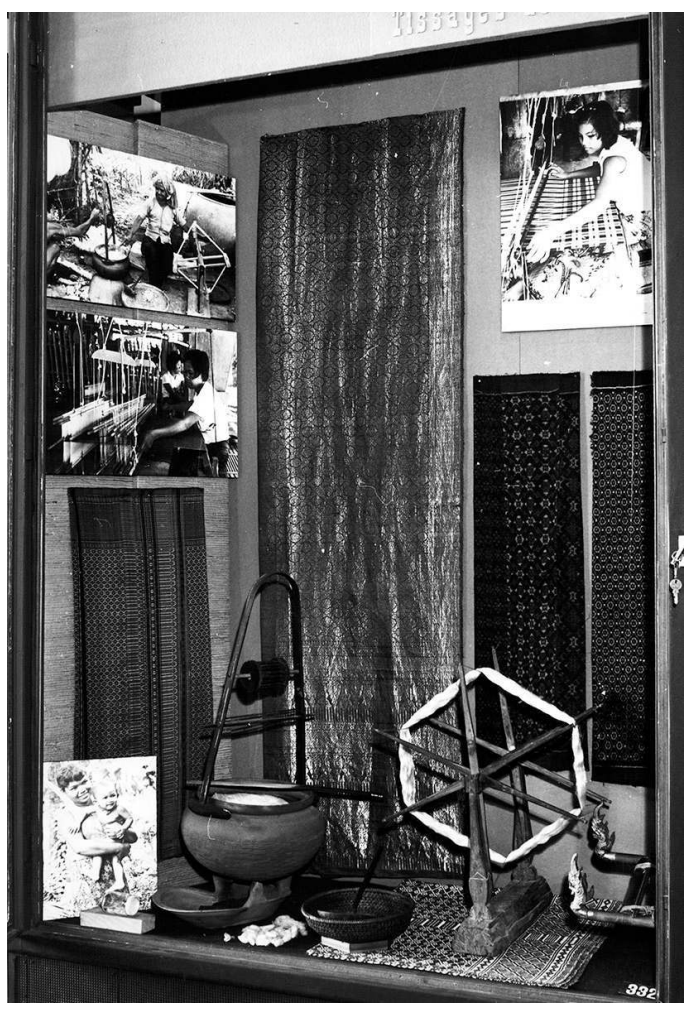

(C) B. Dupaigne

Figure 14 : Vitrine du Musée de l'Homme. Divers accessoires de tissage, 1982

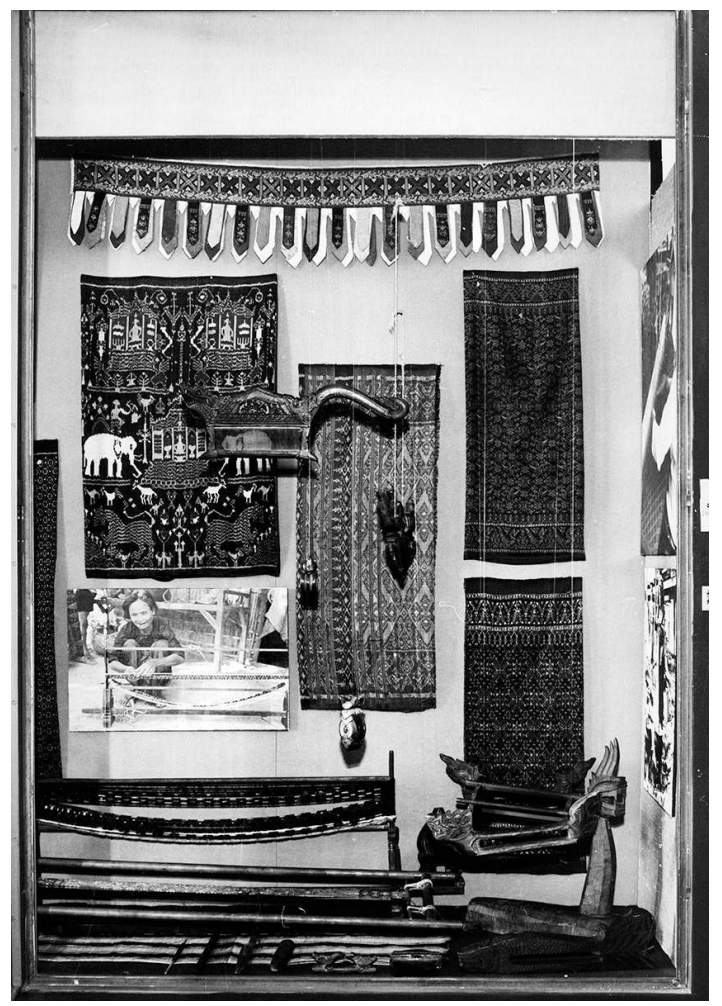

(C) B. Dupaigne 


\section{BIBLIOGRAPHIE}

Aubaile-Sallenave F. 1987 - Bois et bateaux du Vietnam Paris, Selaf, 182 p. (Ethnosciences ; 3).

Aymonier É. 1885 - Notes sur le Laos. Exc. et Recon., IX, 22, mars-avril.

Aymonier É. 1900 - Le Cambodge. Le royaume actuel, I. Ernest Leroux.

Blandin M. 1910 - Le mackloeu (arbre à teinture). Bulletin économique de l'Indochine 86 : 604.

Bouinais A. \& Paulus A. 1885 - L'Indochine française contemporaine, 1, Cochinchine, Cambodge. Challamel aîné, Librairie maritime et coloniale, $2^{\mathrm{e}}$ éd.

Brenier H. 1930 - L'Indochine économique. In : Maspero G. (Ed.) Un empire colonial français, L'Indochine II.

Capus G. 1930 - Les Produits Coloniaux d'origine végétale. Paris, Librairie Larose, 500 p. (Les manuels coloniaux).

Cardon D. 1990 - Guide des teintures naturelles : plantes, lichens, champignons, mollusques et insectes. Paris, Delachaux \& Niestlé, 400 p.

Crevost C. \& Lemarié C. 1921-Catalogue des produits de l'Indochine, t. II. Plantes et produits filamenteux et textiles. Hanoi, Gouvernement général de l'Indochine, Service économique, $290 \mathrm{p}$.

Delvert J. 1961 - Le paysan cambodgien. Paris, Mouton, 740 p.

Dupaigne B. 1980 - Répartition des tissages traditionnels au Cambodge. In : Cheminements. Écrits offerts à Georges Condominas, Asie du Sud-Est et Monde Indonésien, XI (1-4) : 327-337, 2 cartes.

Dupaigne B. 1984 - L'élevage des vers à soie au Cambodge ASEMI, nº spécial « Cambodge II ». Paris, CEDRASEMI, XV (1-4) : 111-131.

Dupaigne B. 2004 - Weaving in Cambodia In: Through the Thread of Time. Southeast Asian Textiles, The James H. W. Thompson Foundation Symposium Papers. Bangkok, River Books : 26-30.

Dy Phon P. 2000 - Plants used in Cambodia. Phnom Penh, Olympic, Cambodian Ministry of Agriculture, $915 \mathrm{p}$.

Girard [L.H.] 1887 - Notice sur le Mac-lua, plante tinctoriale. Bulletin de la Société des Études Indochinoises $1: 23-24$.

Gosselin C. 1900 - Le Laos et le Protectorat français. Paris, Perrin, 349 p.

Harmand J. 1876 - Voyage au Cambodge. Bull. Société de Géographie, oct. : 341-342.

Josselme J. 1883 - Rapport sur la culture du roucou Bulletin de la Société d'Études Indochinoises, $2^{\mathrm{e}}$ trim. : 73.

Klos 1896 - Note sur les principaux articles d'exportation de la Cochinchine et du Cambodge. Bulletin de la Société d'Études Indochinoises 2.

Léonard J. 1881 - Bull. Comité agricole et industriel de Cochinchine : 47.

Leti M., Hul S., Fouché J.G., Cheng S.K., David Bruno 2013 - Flore photographique du Cambodge. Toulouse, Privat 592 p.

Martin M.-A. 1971 - Introduction à l'ethnobotanique du Cambodge. Paris, CNRS, 258 p. +20 pl. photos. 
Martin de Flacourt E. 1927 - Le stick-lac au Cambodge. Bulletin économique de l'Indochine 30 : 115-126.

Maspero G. 1929-1930 - Un empire colonial français, L'Indochine. Paris, Bruxelles, G. Van Oest, 2 t. Morizon R. 1931 - Monographie du Cambodge. Hanoï, Imprimerie d'Extrême orient, 284 p.

Morizon R. 1936 - La province cambodgienne de Pursat. Paris, Ed. Internationales, 198 p.

Morimoto K. 1995 - Silk Production and Marketing in Cambodia. Research Report for Unesco Cambodia, Revival of Silk Weaving Project (14 janvier-14 mai 1995).

Moura J. 1882 - Le Royaume du Cambodge. II. Bulletin de la Société de Géographie Commerciale de Bordeaux : 337.

Nietzki R. 1901 - Chimie des matières colorantes organiques. Paris, G. Carré et C. Naud, 450 p.

Pavie A. 1884 - Cochinchine française. Excursion dans le Cambodge et le royaume de Siam. Saïgon, Imprimerie du gouvernement, $166 \mathrm{p}$.

Raquez A. 1906 - Voyage au Laos. Bulletin de la Société de géographie de Marseille.

Sek You Oun 1973 - Tissage traditionnel des Hol cambodgiens, Phnom-Penh, Faculté d'Archéologie, multigraphié, $104 \mathrm{p}$.

Stoeckel J. 1921-1923 - Étude sur le tissage au Cambodge Arts et Archéologie khmère I (4) : 387-402.

Teston E. \& Percheron M. 1931 - L'Indochine moderne. Encyclopédie administrative, touristique, artistique et économique. Librairie de France, $1032 \mathrm{p}$.

Teulieres R. 1962 - La sériciculture au Sud Viêtnam. Bull. Société des Études indochinoises, XXXVII (l) : 7-36.

Vidal J., Martel G., Lewitz S. 1969 - Notes ethnobotaniques sur quelques plantes en usage au Cambodge. BÉFEO LV (1) : 171-232.

\section{ANNEXES}

Index des termes botaniques

Acacia sp. daem sombueor

Aganonerion polymorphum thnong

Amaranthus spinosus, amaranthe phti

Artocarpus integrifolia, jacquier sauvage khnao prey

Averrhoa bilimb daem trâlung thung

Bombax malabaricum, kapokier sambork kô

Bruguiera gymnorrhiza smâch chroluok

Caesalpinia sappan daem sbaeng,

Combretum quadrangulare sangkae

Corypha Lecomtei, latanier daem treang

Curcuma longa, safran des Indes daem romiet 
Dalbergia nigrescens daem snuol

Diospyros mollis daem makloeua

Ficus benjamina daem chrey krem

Garcinia merguensis daem sandân

Garcinia vilersiana prahout

Hymenocardia Wallichii daem phnum phneng

Indigofera tinctoria, indigotier daem trôm

Lagerstroemia floribunda, goyavier sauvage trobaek prey

Mangifera indica, manguier svay

Melanorrhoea laccifera, arbre à laque daem krêl

Memecylon edule daem phlong

Nauclea brunosis daem khtum

Piper nigrum, poivre mrec

Schleichera triguda daem pongrô

Shorea Cochinchinensis daem propél msau

Sindora cochinchinensis daem krokoh

Tamarindus indica, tamarinier daem ampeul

Zizyphus jujuba, jujubier indien daem putria

\section{NOTES}

1. L'Amaranthus spinosus L., « épinard piquant », daem phtî bonlâ, et l'écorce du fruit chek namva.

2. « Pour une livre de trame, on emploie une livre d'alun », Phnom-Penh, Commission des Mœurs et Coutumes, 80.044.

3. C'est la seule teinture végétale encore utilisée au village de Lovea, près de Siemreap. (Stoeckel 1921-1923 : 397, Vidal et al. 1969 : 209).

4. Le gouverneur khmer de la province de Sâmbaur au nord de Kratié sur le Mékong «percevait une taxe sur les embarcations descendant du Laos, et, chaque année, il portait au roi une redevance traditionnelle de riz, cire, soie, et cœur de jacquier pour la teinture » (Aymonier 1900 : 302).

5. D'autres teintures jaunes sont citées : Gardenia grandifolia, méas dey. Fruits broyés et mis en solution avec citron ou fruits acides, traling tum ou sandam (Stoekel 1921-1923 : 397). Feuilles de palétuvier, Brugmera gymnorrhea, dans la région de Kampot (Stoekel 1921-1923 : 397, Maspero 1929 : 159). Bananiers, Musa sapientum (Morimoto 1995).

6. D'autres plantes ont été utilisées comme teinture jaune : le Morus tinctoria (Moraceae, « mûrier des teinturiers »), la gaude, Reseda luteola, dont toute la plante est utilisée, l'écorce de manguier, svay, Mangifera indica. Le phca-dacam donne une teinture jaune (Bouinais \& Paulus 1885 : 534).

7. À Kompong-Cham. (Teston \& Percheron : 927, Stoeckel 1921-1923 : 398). Sramar, Terminalia chebula, peut-être la variété nana: su mao techek, petit arbre de 3 à $5 \mathrm{~m}$, les fruits sont les myrobolans du commerce, l'un des meilleurs agents tanniques d'Inde. Mélangé avec de l'alun, il 
donne une teinture jaune et avec de l'argile ferrugineuse, une teinture noire (Crévost \& Lemarié $1928: 174)$.

8. Dans les provinces de Kandal, Takeo, Kompong-Speu, Kompong-Thom, Pursat et Battambang (Sek 1973 : 28) et en Trapeang Srang, Kirivong. (Martin de Flacourt 1927).

9. À La-Pho, huyên de Thanh-Thuy, Guide G.B. Indochine, Saïgon, 1927, p. 94.

10. 48 tonnes 470 en ont été exportés en France en 1930. Compagnie "La Gomme Laque» installée à Hanoï et 41 boulevard Magenta, à Paris, (Teston \& Percheron $1931: 783$ et note 775).

11. Sur les cochenilles à laque, voir Cardon (1990:378-381).

12. Famille des Combretaceae. «Très nombreux dans le srok de Bati » (Delvert $1961: 430$ ).

13. Dans le srok de Kandal Stung, en Kandal, les diguettes sont encore plantées d'arbres serrés, formant haie. Ce sont surtout des sangker, des tréang, des chrey : «la région était, il y a vingt ans, productrice de stick-laque que des cochenilles tirent de cet arbre. Cette activité a aujourd'hui disparu, mais le paysage en porte encore fortement la trace ... Le sangker a été l'objet d'une exploitation active pour la fabrication du stick laque dans une région très peuplée (180-200 habitants au $\mathrm{km}^{2}$ ), et dont les ressources sont limitées (rizières généralement très pauvres) » (Delvert $1961:$ 326-327).

14. Dicton relevé par Sek You Oun (1973: 30).

15. Phnom-Penh, Commission des Mœurs et Coutumes, Lovea Em, Kandal, $\mathrm{n}^{\circ}$ 80.022, 1952.

16. Au Laos, le mordant décrit est "de l'eau acidulée, au moyen de vinaigre ou de citron » (Gosselin 1900 : 255).

17. Pour teindre une livre de soie, il faut 8 livres de stick-lac. L'alun comme mordant prépare les fibres à recevoir le colorant. Il faut dix morceaux d'alun pour une livre de soie. On ajoute des mordants naturels, qui rendent les couleurs plus fraîches, mcur prei : des feuilles de con kô, des feuilles de tamarinier acide, Commission des Mœurs et Coutumes, 80.044.

18. J. Léonard (1881 :47), J. Josselme (1883 73), Bouinais \& Paulus (1885 : 335).

19. Le rocouyer était utilisé autrefois à Prek Kdam.

20. «avec de l'eau de chaux» (Martin 1971: 96), Kompong Tralach, Kompong-Thom, Chhlong, Takeo, Tonleap (Stoekel 1921-1923 : 397). On les utilise à Prek Kdam pour teindre les tiges pour les nattes en rouge.

21. Probablement Syzygium Zeylanicum (Myrtaceae).

22. Commission des Mœurs et Coutumes, srok Prei Chor, Kompong-Cham, $n^{\circ} 80.042,1952$

23. Sek (1973 : 30) d'après Nietzki (1901: 403). C'est de cette plante tinctoriale dont doit parler Pierre Poivre dans son Voyage en Cochinchine en 1749-1750. M. Martin (1971: 109) parle du daem kondaol, Careya sphaerica Myrtaceae, une écorce qui donne une teinture rouge brun.

24. Communication de M. Cazaux sur l'indigo du Cambodge. «M. Vouët fait observer que la ferme des Mares fabrique aujourd'hui, en petite quantité, de l'indigo de qualité supérieure probablement à celui qu'envoie M. Cazaux », Séance du Comité agricole et industriel du 13 septembre 1882, BSEI, $1^{\text {er }}$ trim. 1883, p. 17. « Cazeaux, planteur d'indigo, à Oknha-tey, Cambodge, membre de la Société », p. 51.

25. Annuaire illustré du Cambodge, Phnom-Penh, 1904, p. 46. "L'indigo donne de bons résultats dans les provinces de Saang et de Ponhéa-Lu. On l'exporte en assez grande quantité ", « Monographie de la Résidence de Kandal (Kompong-Speu)», BSEI, 1916-1917, p. 213-256.

26. L. Cazeaux, sous-directeur de la Ferme des Mares, «Rapport sur l'indigo cultivé et préparé à la ferme des Mares ", Bulletin du Comité agricole de Cochinchine, IV e série, I, 2, p. 66-80, 1882.

27. «La fabrication de l'indigo ", L'Indépendant de Saïgon. Journal des Chambres de Commerce, 1883, p. 245. Bouinais \& Paulus $(1885: 334,375-376,534)$. A. Cassier, « Culture et préparation de l'indigo au Cambodge », Bulletin économique de l'Indochine, 23-29, 1900. Teston \& Percheron (1931: 928 et 944).

28. «On en trouve principalement dans la province de Kandal, les terres grises argilo-siliceuses lui conviennent parfaitement» (Morizon 1931). 
29. «Les branches sont coupées trois fois dans l'année (ou deux fois seulement si le terrain est inondé), ce qui donne 32 à 68 tonnes de tiges à l'hectare. Elles sont mises à macérer dans des cuves, à raison de 150 kilogrammes de tiges pour 180 litres d'eau; après 24 heures de fermentation, de la chaux est ajoutée : l'indigotine précipitée, l'ensemble est décanté dans une tranchée » (Delvert : 145 et n. 95).

30. Les feuilles de trôm sont laissées à tremper deux ou trois nuits dans une grande jarre remplie d'eau. On en remue le contenu avec un bâton, ce qui trouble le liquide. Puis on transvase le liquide dans une autre jarre, en se gardant de faire couler en même temps les feuilles d'indigo. Au bout d'un moment, les parties denses se déposent au fond, et reste au-dessus le liquide décanté. La partie épaisse au fond de la jarre est le mor. Dans ce mor, on ajoute de la chaux blanche, du sucre et de la soude, tuk kbon. On agite le mélange jusqu'à voir une écume épaisse monter à la surface ; ce mélange est appelé thlah, ce sera le colorant : Commission des Mœurs et Coutumes, srok Prei Chor, Kompong Cham, $\mathrm{n}^{\circ} 80.042$, 1952. Même description du travail pour le Lao chez le Capitaine Gosselin (1900 : 254).

31. L'indigotier est cultivé notamment dans le srok de Muk Kompul, khum de Bak Kheng (province de Kandal), dans l'île de Koh-Sautin et en Peam Chikang, srok de Kang Meas (province de Kompong-Cham).

32. Vu chez les Chams de Koh Sautin, avril 1969.

33. « Pour la teinture, on transforme l'indigo bleu en indigo blanc à l'aide d'un alcali » (Capus $1930: 353$, Sek $1973: 25)$.

34. Pour obtenir du bleu clair, on plonge l'écheveau teint en indigo dans de l'eau contenant du gingembre pilé et du jus de citron ou d'un autre produit acide (Raquez 1906, Stoekel 1921-1923 : 398). Pour les procédés de teinture à l'indigo, comme pour les autres d'ailleurs, voir Cardon 1990).

35. On peut utiliser également une décoction filtrée des écorces et feuilles de daem lovieng, Nerprun tinctoria (Stoekel 1921-1923 : 398, Sek 1973 : 26).

36. «Le mackloeu se multiplie par graines, rejets et marcottes. Il produit au bout de huit ans et est en pleine production à 12 ans; il fleurit de mars à juillet; les fruits sont mûrs de mai à septembre. La récolte se poursuit jusqu'en janvier ; elle consiste à tailler les branches au coupecoupe ». La récolte est extrêmement variable : elle est donnée de 28 à 137 kg par arbre (Delvert $1961: 146)$.

37. À Prek Tameak, le travail est fait par des paysans qui travaillent à façon pour des commerçants de Phnom-Penh. Ils achètent les fruits à leurs voisins et travaillent avec leur famille et des salariés. La teinture occupe en mai 1956, 100 personnes à Prek Tameak (phum Svay At) et 100 autres à Prek Prasap. C'est une activité assez rémunératrice : la teinture de $20 \mathrm{~m}$ de tissu rapporte 100 riels et quatre personnes peuvent teindre 100 pièces de $20 \mathrm{~m}$ en 20 jours, ce qui leur rapporte 10000 riels dont il faut défalquer le prix des fruits (Delvert 1961 : 147). Même description du travail pour le Laos chez le Capitaine Gosselin (1900 : 254).

38. Phum Svay Tani, khum Prek Koy, srok Saang, sur la route de Phnom-Penh à Koh Thom.

39. Commission des Mœurs et Coutumes, srok de Prei Chor, Kompong Cham, $n^{\circ} 80.042,1952$.

40. Écorce de smié, Bruguiera gymnorhizae, pour la teinture brune (Pavie 1881: 125, Teston \& Percheron : 927 (région de Kampot), Stoeckel 1921-1923 : 398). Dy Phon : 149 : smae, palétuvier Ceriops taga. Écorce brune kralan pour les filets de pêche (Vidal et al. 1969 : 184, 187). Également, écorce smac dom, pour les filets de pêche en fibres de ramie, Syzygium zeylanicum, daem smach daom (Martin 1971 : 112, Morizon 1936, Vidal et al. $1969:$ 191). 


\section{RÉSUMÉS}

À partir de notes prises lors de visites dans de nombreux villages du Cambodge à la fin des années 60 , l'auteur explique comment les villageois teignaient alors de manière traditionnelle les écheveaux de fils de soie ou de coton, d'étoffes, dans les couleurs suivantes : jaune, rouge, indigo, vert, noir. La plupart des teintes sont obtenues à partir de plantes. Seul le rouge est obtenu à partir d'un produit animal, l'exsudat formé sur des branches d'arbre par des essaims d'insectes parasites, entretenus à cet effet. Pour chaque teinte, on trouve aussi bien des informations détaillées sur la collecte des produits de base, aussi bien les colorants que les adjuvants (mordants), la préparation de la teinture et les diverses étapes du travail du teinturier (proportions, temps, etc.).

Based on the notes he has taken during his trips to many Cambodian villages in the late sixties, the author explains how villagers would use a traditional technique to dye skeins of silk threads, cotton and other fabrics in yellow, red, indigo, green and black. Most of the tints are obtained from plants. Red is the only one obtained from an animal product - exudate that comes from swarms of insect parasites kept on tree branches for this very purpose. Each tint holds detailed information about the collection of basic products, tints as well as additives (mordant), but also on how they made the tints and the various steps of the dyer's work (measurements, times, etc.).

\section{INDEX}

Index géographique : Cambodge, Laos, Vietnam

Mots-clés : plantes tinctoriales, soie, coton, mordant, tisserand, couleurs, indigo

Keywords : dye plants, dye fixative, cotton, weaver, silk, colors, indigo dye

\section{AUTEUR}

\section{BERNARD DUPAIGNE}

Professeur honoraire, Muséum national d'Histoire naturelle, UMR Eco-anthropologie et ethnobiologie 